\title{
UNBOUNDED NORMAL OPERATORS IN HILBERT SPACE \\ BY
}

\section{S. KANIEL(1)}

\section{INTRODUCTION}

The theory of abstract unbounded operators and their extensions developed along two main lines. The first is the theory of extensions of symmetric operators (Achiezer and Glasman [3]). In this case the problem is to extend a symmetric operator to a self-adjoint one. This theory includes necessary and sufficient conditions for the existence of such extensions, as well as complete characterization of the nature of these extensions, especially the nature of the spectrum of various extensions pertaining to the same operator.

This theory imposes severe conditions and can be applied only in particular cases. In order to deal with abstract problems arising in the theory of partial differential equations and integral equations a relaxation is needed. The notions of "generalized solution," "weak solution," etc. (for example in Lax [1]) and the theory of distributions lead to the following problem of extension of general abstract operators. (For the sake of simplicity the illustration below will be confined to operators acting in Hilbert space.)

The operator (differential, integral, etc.) $\mathscr{T}$ is embedded in a certain space $E$. In this space a "natural" domain $\widetilde{D}_{0}$ is considered. Denote $\mathscr{T}$ acting on $\widetilde{D}_{0}$ by $\tilde{T}_{0}$. $\tilde{T}_{0}$ is in general bounded below on $\tilde{D}_{0}$ so it may be closed to an operator $T_{0} \supset \widetilde{T}_{0}$ acting on $D_{0} \supset \tilde{D}_{0}$. By this process the "generalized solutions" are obtained.

In most cases the range of $T_{0}$ is not the whole space so an extension is needed. Among all possible extensions only the "weak solutions" are considered. They are obtained by looking at a natural "formal adjoint" $\mathscr{T}^{*}$ defined on the closure of the set $\tilde{D}_{0}$. Denote this operator by $T_{1}^{*}$ and denote $\left(T_{1}^{*}\right)^{*}$ by $T_{1}$. Any function in the domain of $T_{1}$ is called a "weak solution." In general the range of $T_{1}$ is the whole space but there are "too many" weak solutions because there are null solutions. So the problem is to find an intermediate $T_{0} \subset T \subset T_{1}$ having a bounded inverse defined on the whole space. Somewhat different versions are in G. C. Rota [4] and Gohberg and Krein [5]. These general theories have only weak results, because of the generality of the operators considered.

Received by the editors September 14, 1962.

(1) This work is a part of a Ph.D. Thesis written under the instruction of Professor S. Agmon. It was also sponsored in part by the National Science Foundation Grant NSF I-19135. 
In this work the author attempts to find "intermediates" between symmetric and general operators; these are the unbounded normal operators.

Unbounded normal operators are defined in Chapter 2. In addition to the commutative relations there are conditions imposed on the domains of a normal operator and its adjoint. Most of this chapter is devoted to theorems dealing with properties of such operators. In Chapter 3 the extensions of normal operators are considered. An important particular case is the complete normal extensions. These have an inverse which is a bounded normal operator. In this case there are theorems investigating the local behaviour of the spectrum, the asymptotic distribution of the eigenvalues, etc. Some theorems on general unbounded operators are obtained in the first chapter.

\section{Chapter 1. General Unbounded Operators}

This chapter which is of introductory type will deal with properties of general (closed) unbounded operators and their extensions. Some of the theorems cited in this chapter are known and will be given without proof except cases where adding the proof is desirable for fluent reading of the subsequent paragraphs.

Throughout this paper: Capital letters $S, T$, etc., will be used to denote operators. The domain of an operator $T$ will be denoted by $D_{T}$, its range by $\Delta_{T}$. Sequences will be denoted by $\left\{f_{n}\right\},\left\{g_{i}\right\}$, etc. Other notations are standard in some basic books (and are not standard in the others).

DEFINITION 1.1. An operator $S$ will be called an extension of an operator $T$ if:

(a) $D_{S} \supset D_{T}$,

(b) if $f \in D_{T}$ then $S f=T f$.

Denote it briefly by $S \supset T(S$ contains $T)$.

Definition 1.2. A point $\lambda$ will be called a regular point of $T$ (or $T$ is regular in the point $\lambda$ ) if there exists a constant $C$ such that:

$$
\|T f-\lambda f\| \geqq C\|f\| \text { for } f \in D_{T} .
$$

The complement of the regular points will be called the spectrum of $T . T$ is regular means: $T$ is regular in zero.

A known theorem is the following: For any closed operator the set of its regular points is open (this theorem will be proved implicitly by Theorem 1.2).

Definition 1.3. $T$ regular in $\lambda$. The deficiency index of $T-\lambda I$ will be the dimension of the orthogonal complement of $\Delta_{T-\lambda I}$. (The deficiency index may be finite or infinite.)

A known theorem asserts that the deficiency index of $T-\lambda I$ is constant (with respect to $\lambda$ ) in any connected component of the set of the regular points of $T$. (This theorem too will be proved by Theorem 1.2.)

A general operator $T$ may be extended in a variety of ways, but the interesting extensionsare obtained as the restriction of an adjoint operator in the following way: 
Definition 1.4. $T_{1}^{*} \subset T^{*}$. $\tilde{T}$ will be called an extension of $T$ with respect to $T_{1}^{*}$ if:

(a) $\tilde{T} \supset T$,

(b) $\tilde{T} \subset\left(T_{1}^{*}\right)^{*}$.

Definition 1.5. $\tilde{T}$ is a regular extension of $T$ if $\tilde{T}$ is regular. $\tilde{T}$ is a complete regular extension if $\Delta_{\tilde{T}}=H$ (the entire space).

Definition 1.6. $T$ is regular in $\lambda$. The greatest constant $C$ satisfying: $\|T f-\lambda f\| \geqq C\|f\|$ for $f \in D_{T}$ will be called the greatest lower bound of $T_{\lambda}$.

LEMMA 1.1. T is a regular operator and is bounded below by $C$. Conclusions:

(a) $\Delta_{T^{*}}=H$.

(b) There exists a unique right inverse $S$ to $T^{*}\left(T^{*} S=I\right)$ bounded from above by $1 / C$ and mapping $H$ into $\Delta_{T}$.

Proof. The theorem as well as the proof can be found in the literature (perhaps in a somewhat different setting). The proof will be given for the sake of completeness.

It is clear that (a) follows from (b). In order to prove (b) define a functional $l_{h}$ acting on functions in $\Delta_{T}$ in the following way: If $\phi=T g$ then $l_{h}(\phi)=(g, h)$ where $h$ is an arbitrary fixed element. This functional is bounded because:

$$
\frac{l_{h}(\phi)}{\|\phi\|}=\frac{(g, h)}{\|\phi\|} \leqq \frac{\|g\| \cdot\|h\|}{\|\phi\|}=\frac{\|g\| \cdot\|h\|}{\|T g\|} \leqq \frac{1}{C}\|h\| .
$$

Therefore $l_{h}(\phi)$ can be represented by a function $f, f \in \Delta_{T}$, satisfying:

$$
l_{h}(\phi)=(\phi, f)=(T g, f)=(g, h)
$$

Hence by the definition of $T^{*}: h=T^{*} f$ and by (1.1) $\|h\| \geqq C\|f\|$. It is clear that the mapping $h \rightarrow f$ is linear; denote this operator by $S$. So by $(1.2)(T g, S h)=(g, h)$ or $T^{*} S=I$. It is evident that $S$ satisfies (b). The uniqueness follows from the unique definition of $f$.

With the aid of this lemma the existence of extensions of a regular operator $T$ with respect to a regular $T_{1}^{*} \subset T^{*}$ can be proved and estimates of the greatest lower bound can be obtained in the following way:

THEOREM 1.1. T and $T_{1}^{*}\left(T_{1}^{*} \subset T^{*}\right)$ are regular operators; $c, c^{*}$ are their greatest lower bounds respectively.

Conclusion: There exists a complete regular extension of $T$ with respect to $T_{1}^{*}$, denote it by $\tilde{T}$ having $\bar{c}=\min \left\{c, c^{*}\right\}$ as its greatest lower bound.

Proof. The idea is to generalize Krein's method of extension of symmetric operators. By the conditions of this theorem $\Delta_{T}$ is a closed subspace and the mapping $R: T f \rightarrow f$ defines a bounded operator from $\Delta_{T}$ into $H$ with $1 / c$ as a bound. Obviously $T R=I$ (on $\Delta_{T}$ ). Extend $R$ boundedly to an operator $\tilde{R}$ in such a way that $\left(T_{1}^{*}\right)^{*} \tilde{R}=I$ (on $\left.H\right)$. By this equality $\widetilde{R}$ has a left inverse; denote it by $\tilde{T}$ which 
satisfies $T \subset \tilde{T} \subset\left(T_{1}^{*}\right)^{*}$. Moreover the lower bound of $\tilde{T}$ is the upper bound of $\tilde{R}$ and $\Delta_{\tilde{T}}=H$.

The extension will be carried by two steps. The first step will be the extension of $P R$ where $P$ denotes the projection operator from $H$ to $\Delta_{T_{1}^{*}}$. The second step will be the extension of $(I-P) R$. Consider Lemma 1.1 for the operator $T_{1}^{*}$. There exists an operator $S$ such that $\left(T_{1}^{*}\right)^{*} S=I$ mapping $H$ into $\Delta_{T_{1}^{*}}$. On the other hand $\left(T_{1}^{*}\right)^{*} P R=I$, because for any $f \in D_{R}\left(f \in \Delta_{T}\right)$ and $g \in D_{T_{1}^{*}}$ :

$$
\left(\left(T_{1}^{*}\right)^{*} P R f, g\right)=\left(P R f, T_{1}^{*} g\right)=\left(R f, T_{1}^{*} g\right)=(T R f, g)=(f, g) .
$$

Hence by the uniqueness of $S$ it follows that $S \supset P R$. Denote now $S$ by $(P R) \sim$ the desired extension of $P R$.

Extend now $(I-P) R$ to $(I-P) R^{\sim}$ in such a way that $\Delta_{(I-P) R^{\sim}}$ will be orthogonal to $\Delta_{T_{i}^{*}}$ and the extended operator will satisfy:

$$
\|\tilde{R} g\|^{2}=\|(P R) \sim g\|^{2}+\left\|(I-P) R^{\sim} g\right\|^{2} \leqq\left[\max \left\{\frac{1}{c}, \frac{1}{c^{*}}\right\}\right]^{2}\|g\|^{2} .
$$

Define the bilinear symmetric form:

$$
[f, g]=\left[\max \left\{\frac{1}{c}, \frac{1}{c^{*}}\right\}\right]^{2}(f, g)-\left((P R) f^{\sim},(P R)^{\sim} g\right) .
$$

By Lemma 1.1, $\|f\|^{2} \cdot\left(1 / c^{*}\right)^{2} \geqq\left((P R)^{\sim} f,(P R)^{\sim} f\right)$; hence $[f, f] \geqq 0$. Define $[[f]]=[f, f]^{1 / 2}$ and observe that (1.3) may be written as:

$$
\left\|(I-P) R^{\sim} f\right\| \leqq[[f]] .
$$

As $[[]]$ is a semi-norm and $(P R)^{\sim}$ is a continuous operator, it follows that the linear manifold of all $g$ satisfying $[[g]]=0$ is a closed subspace $K$. Therefore the quotient space $H / K$ can be defined, by assigning [ [ ]] as its norm and [ ] as its scalar product. Complete this space to a Hilbert space $\hat{H}$.

The inequality $\|(I-P) R f\| \leqq[[f]]$ is valid because $\|P R f\|^{2}+\|(I-P) R f\|^{2}$ $=\|R f\|^{2} \leqq\left(1 / c^{2}\right)\|f\|^{2}$. Therefore by the mapping $H \rightarrow \hat{H}$ the operator $(I-P) R$ is mapped into a continuous operator defined on the image of $\Delta_{T}$ and having its range in the image of the orthogonal complement of $\Delta_{T_{1}^{*}}$. Extend now this operator to $\hat{H}$ keeping its norm fixed and keeping its range in the image of $\Delta_{T_{i}^{*}}$. This extension induces extension of the original operator $(I-P) R$ to $(I-\widetilde{P}) R$ which satisfies (1.5). Hence $\widetilde{R}=(P R)^{\sim}+(I-\widetilde{P}) R$ satisfies (1.3), the desired inequality.

For applications in the next chapters it is important to know the dependence of $\Delta_{T-\lambda I}$ on $\lambda$. In certain cases it is more convenient to investigate the dependence of the orthogonal complement to the range.

The following theorem describes the nature of dependence in case that $T$ has a finite deficiency index.

THEOREM 1.2. T is regular in $\bar{\lambda}_{0}$ and has a finite deficiency index $n . f_{1}, \cdots, f_{n}$ are $n$ orthonormal functions which span $\left[\Delta_{T-\lambda_{0} I}\right]^{+}$(the orthogonal complement). 
In this case there exist analytic functions in a neighborhood of $\lambda_{0}$ (from the complex plane to the space) $f_{1}(\lambda), f_{2}(\lambda), \cdots, f_{n}(\lambda)$ which span $\left[\Delta_{T-\lambda_{I}}\right]^{+}$and $f_{i}\left(\lambda_{0}\right)=f_{i}, i=1,2, \cdots, n$.

Proof. Without loss of generality it is sufficient to consider the case where $\lambda_{0}=0$. Denote $T^{-1}$ by $R^{\prime}$ (defined only on $\Delta_{T}$ ) and extend $R^{\prime}$ boundedly to an operator $R$ defined in the entire space. Consider its adjoint $R^{*}$ and the expression:

$$
f_{i}(\lambda)=f_{i}+\lambda R^{*} f_{i}+\lambda^{2} R^{* 2} f_{i}+\cdots+\lambda^{j} R^{* j} f_{i}+\cdots ;
$$

this is a power series of $\lambda$ which converge in the neighborhood of 0 and the radius of convergence is at least $1 /\left\|R^{*}\right\|$.

For any $h \in D_{T^{*}}$

$$
\begin{aligned}
\left(f_{i}(\lambda), T h-\bar{\lambda} h\right) & =\left(f_{i}(\lambda), T h\right)-\lambda\left(f_{i}(\lambda), h\right) \\
& =\sum_{j=0}^{\infty}\left(\lambda^{j} R^{* j} f_{i}, T h\right)-\lambda \sum_{j=0}^{\infty}\left(\lambda^{j} R^{* j} f_{i}, h\right) \\
& =\left(f_{i}, T h\right)+\lambda \sum_{j=1}^{\infty}\left(\lambda^{j-1} R^{* j-1} f_{i}, R T h\right)-\lambda \sum_{j=0}^{\infty}\left(\lambda^{j} R^{* j} f_{i}, h\right) \\
& =\left(f_{i}, T h\right)=0 .
\end{aligned}
$$

This equality means that the analytic functions defined by (1.6) are orthogonal to $\Delta_{T-\bar{\lambda}_{1}}$.

Moreover,in a small neighborhood of 0 these functions are independent for any $\lambda$. Assume that for some $\lambda_{0} f_{i}\left(\lambda_{0}\right)$ are dependent. It means that there exist constants $\alpha_{i}$ such that $\sum_{i=1}^{n} \alpha_{i} f_{i}\left(\lambda_{0}\right)=0$; and $\alpha_{i}$ may be normalized so that $\alpha_{1}=1$, $\left|\alpha_{i}\right| \leqq 1$.

Take scalar product with $f_{1}$ :

$$
0=\sum_{i=1}^{n}\left(\alpha_{i} f_{i}\left(\lambda_{0}\right), f_{1}\right)=1+\sum_{i=2}^{\infty} \alpha_{i}\left(f_{i}, f_{0}\right)+\sum_{j=1}^{\infty} \lambda_{0}^{j} \sum_{i=1}^{n} \alpha_{i}\left(R^{* J} f_{i}, f_{1}\right) .
$$

The $f_{i}$ form an orthonormal system; therefore the second summand is zero and for $\left|\lambda_{0}\right|<1 /\left\|R^{*}\right\| \cdot 3 n$

$$
\left|\sum_{j=1}^{\infty} \lambda_{0}^{j} \sum_{i=1}^{n} \alpha_{i}\left(R^{* j} f_{i}, f_{1}\right)\right| \leqq \sum_{j=1}^{\infty}\left(\frac{1}{\left\|R^{*}\right\| \cdot 3 n}\right)^{j} \cdot n\left\|R^{*}\right\|^{j} \leqq \frac{2}{3},
$$

so the right-hand side of (1.7) is greater than $1 / 3$.

Because the deficiency index is constant in the neighborhood of 0 the $f_{i}(\lambda)$ $\operatorname{span}\left(\Delta_{T-\lambda_{I}}\right)^{+}$.

REMARKS. (a) The constancy of the deficiency index is implicitly proved. As a consequence of the independence of $f_{i}(\lambda)$ it follows that the deficiency index in a neighborhood of 0 is greater than or equal to that in 0 , and the equality is obtained by "going back" and expanding about a point close to 0 . 
(b) This theorem is true also in case of infinite deficiency index by application of Paley-Wiener theorem on biorthogonal system. As this case is not required in the sequel the proof is omitted.

\section{Chapter 2. Normal Operators}

As was mentioned in the introduction, an "intermediate class" between general closed operators and symmetric operators will be introduced. In Chapter 1 the idea of restriction of the adjoint was employed as, for example, in Lemma 1.1. In this chapter a somewhat more complicated structure will be defined in the following way.

Consider an operator $T_{0}$ defined on a dense manifold; consider its adjoint $T_{0}^{*}$ and a restriction $T_{1}^{*} \subset T_{0}^{*}$ and form the adjoint operator $\left(T_{1}^{*}\right)^{*}$. $\left(T_{1}^{*}\right.$ has to be defined on a dense manifold.) Denote $\left(T_{1}^{*}\right)^{*}$ by $T_{1}: T_{1} \supset T_{0}$.

Using these notations the spaces $H_{\lambda}$ and $H_{\lambda}^{*}$ will be introduced. $H_{\lambda}$ is defined as the subspace of all $f$ satisfying $T_{1} f=\lambda f$; this is the orthogonal complement to $\Delta_{T_{1}^{*}-\bar{\lambda}_{I}}$. Similiarly, $H_{\lambda}^{*}$ is the subspace of all $g$ satisfying $T_{0}^{*} g=\lambda g$, and consequently $H_{\lambda}^{*}$ is orthogonal to $\Delta_{T_{0}-\bar{\lambda}_{I}}$.

In order to define the normal operators additional conditions are required.

Definition 2.1. $\left(T_{0}, T_{1}^{*}\right)$ form a normal pair if:

(a) $T_{0}$ commutes with $T_{1}^{*}$, i.e., if $T_{0} f=g, T_{1}^{*} g=h$ then $f \in D_{T_{1}^{*}} ; T_{1}^{*} f \in D_{T_{0}}$ and $T_{0} T_{1}^{*} f=T_{1}^{*} T_{0} f$.

(b) The closure of $D_{T_{i}^{*} T_{0}}$ with respect to $T_{0}$ norm is $D_{T_{0}}$; i.e. for any $g \in D_{T_{0}}$ there exists a sequence $\left\{g_{i}\right\} \in D_{T_{1}^{*} T_{0}}$ such that $g_{i} \rightarrow g$ and $T_{0} g_{i} \rightarrow T_{0} g$. The same property holds for $T_{1}^{*}$ as well.

(c) $D_{T_{0}^{*}} \cap H_{\lambda}$ is dense in $H_{\lambda}$ and $D_{T_{1}} \cap H_{\lambda}^{*}$ is dense in $H_{\lambda}^{*}$.

$T_{0}$ is normal if $T_{0}$ has a mate $T_{1}^{*}$ such that $\left(T_{0}, T_{1}^{*}\right)$ form a normal pair.

This definition is quite restrictive. It seems that condition (a) alone will not furnish strong results in the case of unbounded operators. However, the author is not convinced that conditions (b) and (c) are the best. They were chosen because many "natural" operators like partial differential operators with constant coefficients satisfy these conditions.

In detail: Take a differential operator with constant coefficients $\mathscr{T}$ and denote its formal adjoint by $\mathscr{T}^{*}$. $T_{0}$ will be defined by restricting $\mathscr{T}$ to the closure of $D$ (the set of functions with compact support). $T_{1}^{*}$ will be $\mathscr{T}^{*}$ restricted to the closure with respect to $\mathscr{T}^{*}$ of $D$.

Property (b) is valid because $D$ is in the domain of $T_{0} T_{1}^{*}$. Property (c) is valid because the exponentials are in the domain of $T_{0}^{*}$ and $T_{1}$ and by a known theorem of Malgrange exponentials are dense in $H_{\lambda}$ and in $H_{\lambda}^{*}$. Observe also that any symmetric operator satisfies (b) and (c) automatically.

In this work most of the results will be obtained for operators having a finite deficiency index. Even in this case the results call for further investigation.

LEMmA 2.1. $T_{0}^{*}$ is invariant in $H_{\lambda}$ and $T_{1}^{*}$ is invariant in $H_{\lambda}^{*}$. 
Proof. If $g \in D_{T_{0}^{*}}$ and $f \in D_{T_{0} T_{i}^{*}}$ then:

$$
\begin{aligned}
\left(T_{0}^{*} g, T_{1}^{*} f\right) & =\left(g, T_{0} T_{1}^{*} f\right)=\left(g, T_{1}^{*} T_{0} f\right)=\left(T_{1} g, T_{0} f\right) \\
& =\left(\lambda g, T_{0} f\right)=\left(\lambda T_{0}^{*} g, f\right)
\end{aligned}
$$

But by property (b) the equality $\left(T_{0}^{*} g, T_{1}^{*} f\right)=\left(\lambda T_{0}^{*} g, f\right)$ is valid for any $f \in D_{T_{1}^{*}}$ which means that $T_{0}^{*} g \in H_{\lambda}$. The same proof holds for the second part.

THEOREM 2.1. $\left(T_{0}, T_{1}^{*}\right)$ form a normal pair. $T_{1}^{*}$ is regular in a region $\bar{D}$ and has a finite deficiency index $n$.

Subject to those conditions there exists an analytic function of two variables $G(Z, \omega)$ :

$$
G(Z, \omega)=\omega^{n}+h_{1}(Z) \omega^{n-1}+\cdots+h_{n}(Z), Z \in D,
$$

having the property: For any $Z_{0}$ the zeros of $G\left(Z_{0}, \omega\right)$ are the eigenvalues of $T_{0}^{*}$ restricted to $H_{\mathrm{Z}_{0}}$.

Proof. In the case where $T_{1}^{*}$ has a finite deficiency index, property (c) of Definition 2.1 may be restated simply as $H_{\lambda} \in D_{T_{0}^{*}}$.

Choose an arbitrary point $\hat{\lambda} \in D$. Denote by $f_{1}, \cdots, f_{n}$ an orthonormal system in $H_{\lambda}$. $T_{1}^{*}$ satisfies the conditions of Theorem 1.2 ; therefore there exist analytic functions $f_{i}(\lambda)$ which span $H_{\lambda}$ satisfying $f_{i}(\hat{\lambda})=f_{i}$. (Observe that $H_{\lambda}$ is the orthogonal complement of $\Delta_{T_{1}^{*}-\lambda_{I}}$.) By normality hypothesis $T_{0}^{*}$ is invariant in $H_{\lambda}$, therefore

$$
T_{0}^{*} f_{i}(\lambda)=\sum_{j=1}^{n} \alpha_{i j}(\lambda) f_{j}(\lambda)
$$

$\alpha_{i j}(\lambda)$ are numerical functions of $\lambda$, moreover they are analytic. The proof is the following: for any $g \in D_{T_{0}}:\left(T_{0}^{*} f_{i}(\lambda), g\right)=\left(f_{i}(\lambda), T_{0} g\right)$; therefore

$$
\sum_{j=1}^{n}\left(\alpha_{i j}(\lambda) f_{j}(\lambda), g\right)=\sum_{j=1}^{n} \alpha_{i j}(\lambda) \cdot\left(f_{j}(\lambda), g\right)=\left(f_{i}(\lambda), T_{0} g\right)
$$

$D_{T_{0}}$ is dense in the space; therefore $g_{1}, g_{2}, \cdots, g_{n} \in D_{T_{0}}$ may be chosen satisfying the following property: The $n \times n$ matrix $\left(f_{j}(\lambda), g_{k}\right)$ is regular in a neighborhood of $\hat{\lambda}$. So $\alpha_{i j}(\lambda)$ solve the system of $n$ linear equations:

$$
\sum_{j=1}^{n} \alpha_{i j}(\lambda) \cdot\left(f_{j}(\lambda), g_{k}\right)=\left(f_{i}(\lambda), T_{0} g_{k}\right), \quad k=1,2, \cdots, n .
$$

Because the right-hand side as well as the coefficients are analytic functions of $\lambda$, the solutions must be analytic.

The eigenvalues of $T_{0}^{*}$ restricted to $H_{\lambda}$ are the zeros of the characteristic polynomial of the matrix $\left(\alpha_{i j}(\lambda)\right)$. Its coefficients are polynomials in the matrix's 
entries; therefore they are analytic functions. So the characteristic polynomial is of the form $\omega^{n}+h_{1}(Z) \omega^{n-1}+\cdots+h_{n}(Z)$ where $h_{i}(Z)$ are analytic in a neighborhood of $\hat{\lambda}$. This is the desired $G(\omega, Z)$.

In order to complete the proof it is necessary to show that $h_{i}(Z)$ are analytic in $D$. As coefficients of the characteristic polynomial they are not dependent on the special point $\hat{\lambda}$ nor on the special choice of $f_{i}(\lambda)$. So there is a unique continuation to the entire domain.

An important special case is when $T_{0}$ and $T_{1}^{*}$ are regular in the entire plane. Differential operators acting on the closure of functions with compact support serve again as an example. The Hörmander inequality [2] states that for any operator $\mathscr{T}$ and any $f \in D:\|\mathscr{T} f\| \geqq C\|f\|$ and $C$ is dependent only on the domain and on the order of the operator. Therefore $\|\mathscr{T} f-\lambda f\| \geqq C\|f\|$ for any $\lambda$, or by the definition of $T_{0}$ and $T_{1}^{*}\left\|T_{0} f-\lambda f\right\| \geqq C\|f\|$ and $\left\|T_{1}^{*} f-\lambda f\right\| \geqq C\|f\| \quad$ (the same $C$ ).

By adding the conditions of finiteness of the deficiency indices the following theorem is obtained.

THEOREM 2.2. $T_{0}, T_{1}^{*}$ form a normal pair, are regular in the entire plane and have finite deficiency indices.

Subject to these conditions there exists a polynomial in two variables $P(Z, \omega)$ having the property: If $P\left(Z_{0}, \omega_{0}\right)=0$ then there exists an eigenfunction $f$ satisfying $T_{1} f=Z_{0} f, T_{0}^{*} f=\omega_{0} f$, and if $f$ is a common eigenfunction with eigenvalues $Z_{0}, \omega_{0}$, then $P\left(Z_{0}, \omega_{0}\right)=0$.

An illustration to the general case will be the proof in the special case where $T_{0}$ and $T_{1}^{*}$ have deficiency index 1 . In this case for each $Z$ there exists only one eigenfunction $f: T_{1} f=Z f$ and for each $\omega$ there exists only one eigenfunction $g: T_{0}^{*} g=g$.

By Lemma 2.1 $T_{1} f=Z f$ implies $T_{0}^{*} f=\mu(Z) f$ and by Theorem $2.1 \mu(Z)$ is analytic function of $Z$ and $G(Z, \omega)=\omega-\mu(Z)$.

Because $T_{1}^{*}$ is regular in the whole plane $\mu(Z)$ is an entire function. $\mu(Z)$ is simple because if $\mu\left(Z_{1}\right)=\mu\left(Z_{2}\right)=\mu$, there are two independent functions $f_{1}, f_{2}$ : $T_{1} f_{1}=Z_{1} f_{1} ; T_{0}^{*} f_{1}=\mu f_{1} ; T_{1} f_{2}=Z_{2} f_{2} ; T_{0}^{*} f_{2}=\mu f_{2}$. Hence $T_{0}$ would have the deficiency index 2 in $\mu$.

An entire simple function is linear, therefore $\mu(Z)=\alpha Z+\beta$ and

$$
P(Z, \omega)=\omega-\alpha Z-\beta .
$$

In the general case the characteristic function

$$
G(Z, \omega)=\omega^{n}+\omega^{n-1} h_{1}(Z)+\cdots+h_{n}(Z)
$$

is a polynomial in $\omega$ with coefficients that are entire functions in $Z$. Fix $\omega\left(\omega=\omega_{0}\right)$ and look at the zeros of the entire function in a single variable 


$$
\tilde{h}(Z)=\omega_{0}^{n}+\omega_{0}^{n-1} h_{1}(Z)+\cdots+h_{n}(Z) .
$$

Any zero $Z_{0}$ of this function corresponds to an eigenfunction $g$ satisfying $T_{0}^{*} g=\omega_{0} g ; T_{1} g=Z_{0} g$.

As the deficiency index of $T_{0}$ is finite only a finite number of $Z$ (which cannot exceed the deficiency index) are zeros of $\tilde{h}(Z)$, because any zero corresponds to an eigenvalue of $T_{1}$ restricted to $H_{\omega_{0}}^{*}$.

Therefore Theorem 2.2 will be established by proving the following statement on functions of two complex variables.

THEOREM 2.3. If $G(z, \omega)$ is entire in $z$ and polynomial in $\omega$ with leading coefficient 1 and has the property: for any $\omega_{0}$ there are at most $m$ zeros of $G\left(z, \omega_{0}\right)$, then $G(z, \omega)$ is polynomial in $z$ and $\omega$.

Proof $\left({ }^{2}\right)$. For any fixed $\omega G(\omega, z)=P_{\omega}(z) \cdot e^{g_{\omega}(z)}$ where $P_{\omega}(z)$ is a polynomial in $z$ and $g_{\omega}(z)$ is entire. Fix $\omega_{i}$ and obtain the system:

$$
\omega_{i}^{n}+h_{1}(z) \omega_{i}^{n-1}+\cdots+h_{n}(z)=P_{i}(z) \cdot e^{g_{i}(z)}, \quad i=1, \cdots, n-1 .
$$

So by solving for $h_{k}(z)$ (with subscripts omitted):

$$
h_{k}(z)=\sum_{i=1}^{h} \alpha_{i k} \cdot P_{i}(z) \cdot e^{g_{i}(z)}+\beta_{k}, \quad \alpha_{i k}, \beta_{k}=\text { constants. }
$$

Fix now another $\omega \neq \omega_{i}$; then:

$$
\omega^{n}+\sum_{k=1}^{n} \omega^{n-k}\left(\sum_{i=1}^{n} \alpha_{i k} P_{i}(z) e^{g_{i}(z)}+\beta_{k}\right)=P_{\omega}(z) e^{g_{\omega}(z)}
$$

Group now terms with the same exponential:

$$
Q_{0}(\omega)+\sum_{s} P_{s}(\omega, z) e^{g_{s}(z)}=P_{\omega}(z) \cdot e^{g_{\omega}(z)}
$$

$Q_{0}(\omega)$ is a nonvanishing polynomial in $\omega$ because its leading coefficient is 1 . $P_{s}(\omega, z)$ are polynomials in $\omega$ and $z$. This equality as a function in $z$ implies $g_{\omega}(z)=0$ for all $\omega$ that are not zeros of $Q_{0}(\omega)$. Furthermore $Q_{0}(\omega)=P_{\omega}(z)$ and $\sum_{s} P_{s}(\omega, z) e^{g_{s}(z)}=0$.

The last equality implies that for any $s$ either $P_{s}(\omega, z)$ or $g_{s}(z)$ are zero identically. In any case the left-hand side of $(2.3)$ (which is $G(z, \omega)$ ) is polynomial in $\omega$ and $z$. Thus the proof of Theorem 2.3 as well as Theorem 2.2 is complete.

Theorem 2.3 does not tell the degree of $G(z, \omega)$ in $\omega$ but by using it again for the operator $T_{0}^{*}$ (instead of $T_{1}$ ) the following theorem is obtained.

THEOREM 2.4. There exists a polynomial $Q(z, \omega)$ which has degree $m$ (the deficiency index of $T_{0}$ ) at most in $z$ and degree $n$ at most in $\omega$ and has leading coefficients 1 in $z$ and $\omega$.

(2) This elementary proof was given by M. Shinbrot. 
Proof. By application of Theorem 2.2 to $T_{0}^{*}$ there exists a polynomial $R(z, \omega)$ :

$$
R(z, \omega)=z^{m}+h_{1}(\omega) z^{m-1}+\cdots+h_{m}(\omega)
$$

having the property that each zero $\left(z_{0}, \omega_{0}\right)$ corresponds to an eigenfunction $f$ : $T_{0}^{*} f=\omega_{0} f, T_{1} f=z_{0} f$, and vice versa. But the polynomial $G(z, \omega)$ has also that property, i.e., that any function $f: T_{1} f=z_{0} f, T_{0}^{*} f=\omega_{0} f$ corresponds to a zero $\left(z_{0}, \omega_{0}\right)$ of $G(z, \omega)$. It follows that the zeros of $G(z, \omega)$ and $R(z, \omega)$ are the same (they may have different multiplicity). Define $Q(z, \omega)$ as the greatest common divisor of $G(z, \omega)$ and $R(z, \omega)$, and factor $G(z, \omega)$ and $R(z, \omega)$ into prime factors:

$$
\begin{aligned}
& G(z, \omega)=Q_{1}^{\alpha_{1}}(z, \omega) \cdot Q_{2}(z, \omega)^{\alpha_{2}} \cdots Q_{n}^{\alpha_{n}}(z, \omega), \\
& R(z, \omega)=Q_{1}^{\beta_{1}}(z, \omega) \cdot Q_{2}(z, \omega)^{\beta_{2}} \cdots Q_{n}^{\beta_{n}}(z, \omega) .
\end{aligned}
$$

$\alpha_{i}$ and $\beta_{i}$ may be zero. $Q(z, \omega)$ is given by:

$$
Q(z, \omega)=Q_{1}^{\min \left[\alpha_{1}, \beta_{1}\right]} \cdot Q_{2}^{\min \left[\alpha_{2}, \beta_{2}\right]} \cdots Q_{n}^{\min \left[\alpha_{n}, \beta_{n}\right]} .
$$

As $G(z, \omega)$ and $R(z, \omega)$ have the same zeros it follows by Hilbert's nullstellensätz that $G(z, \omega)$ divides a power of $R(z, \omega)$ and vice versa. Therefore if for any $i \alpha_{i}>0$ then $\beta_{i}>0$ and $\min \left(\alpha_{i}, \beta_{i}\right)>0$. Hence $Q(z, \omega)$ has the same zeros as $G(z, \omega)$ and $R(z, \omega)$. As $Q(z, \omega)$ divides $G(z, \omega)$ and the leading coefficient in $\omega$ of $G(z, \omega)$ is 1 it is clear that the leading coefficient in $\omega$ of $Q(z, \omega)$ is 1 . The same argument holds for $z$; therefore $Q(z, \omega)$ is the desired polynomial.

CoROllary 2.4. $Q(z, \omega)$ can be computed by knowing a finite number of pairs of eigenvalues pertaining to the common eigenfunctions of $T_{1}$ and $T_{0}^{*}$.

Because by Theorem 2.4, the degree of $Q(z, \omega)$ is bounded in $z$ and $\omega$. Hence its coefficients can be computed by knowing a finite number of zeros.

So far nothing was mentioned about existence of normal operators that are not derived from symmetric operators. The theory of symmetric operators and their extensions is quite complete and may be extended to polynomials in symmetric operators as well. Therefore it is necessary to furnish an example of a normal operator which is not a polynomial in a symmetric operator. The example will also show the structure of a normal operator which is regular in the whole plane; observe in particular the use of Theorem 2.3 as a guide for construction of the eigenvalues.

EXAMPLE. There exists a normal pair of operators $\left(T_{0}, T_{1}^{*}\right)$ which are regular in the whole plane. Moreover, there exists an operator $T$, extension of $T_{0}$, such that: $T \supset T_{0} ; T^{*} \supset T_{1}^{*}$; and $R=T^{-1}$ is a bounded normal operator. $T$ as well as $R$ are not polynomials in any symmetric operator. This example is quite complicated. Because of reasons that will be explained in Chapter 3 the deficiency index of $T_{0}$ and $T_{1}^{*}$ is 2 at least. It is more convenient to construct $R$ and $R^{*}$ first, then $T$ and $T^{*}$, and finally $T_{0}$ and $T_{1}^{*}$. 
Choose any orthogonal sequence $\left\{f_{n}\right\}$ in a separable Hilbert space $H$ and define

$$
\begin{aligned}
R f_{n} & =\frac{1}{\lambda_{n}} f_{n} ; & & R^{*} f_{n}=\frac{1}{\lambda_{n}} f_{n} ; \\
\left(\operatorname{Re} \lambda_{n}\right)^{2}-\left(\operatorname{Im} \lambda_{n}\right)^{2} & =\frac{1}{2} ; & & \left|\lambda_{n}\right| \rightarrow \infty .
\end{aligned}
$$

Further conditions will be imposed later on the $\lambda_{n}$. By this definition $R$ and $R^{*}$ are bounded normal operators having well-defined inverses $T, T^{*}$ respectively:

$$
T f_{n}=\lambda_{n} f_{n} ; \quad T^{*} f_{n}=\bar{\lambda}_{n} f_{n} .
$$

The function $g=\sum_{j=0}^{\infty} d_{j} f_{j}$ is in the domain of $T\left(T^{*}\right)$ if and only if $\sum_{j=0}^{\infty}\left|d_{j} \lambda_{j}\right|^{2}<\infty . D_{T}=D_{T^{*}}$ is dense in $H$ and $\Delta_{T}=\Delta_{T^{*}}=H . T_{0}$ and $T_{1}^{*}$ will be defined by "taking out" two functions $\sum_{j=0}^{\infty} a_{j} f_{j}$ and $\sum_{j=0}^{\infty} b_{j} f_{j}$ from the range of $T$.

They are chosen to satisfy the following conditions:

$$
\begin{aligned}
& \sum_{j=0}^{\infty}\left|a_{j}\right|^{2}=\sum_{j=0}^{\infty}\left|b_{j}\right|^{2}=1 ; \quad \sum_{j=0}^{\infty} a_{j} b_{j}=0 . \\
& a_{n}\left(\frac{\lambda_{n}}{\bar{\lambda}_{n}}-\frac{1}{\bar{\lambda}_{n}}\right)+b_{n}=0 . \\
& -a_{n}+b_{n}\left(\frac{\lambda_{n}}{\bar{\lambda}_{n}}+\frac{1}{\bar{\lambda}_{n}}\right)=0 .
\end{aligned}
$$

(2.8) Any linear combination of the series $\left\{\lambda_{n} a_{n}\right\}$ and $\left\{\bar{\lambda}_{n} b_{n}\right\}$ is not square integrable. A necessary and sufficient condition for existence of solutions for (2.6) and (2.7) is:

$$
\left(\frac{\lambda_{n}}{\bar{\lambda}_{n}}-\frac{1}{\bar{\lambda}_{n}}\right)\left(\frac{\lambda_{n}}{\bar{\lambda}_{n}}+\frac{1}{\bar{\lambda}_{n}}\right)+1=0
$$

which by evaluation reduces to (2.4).

Take for the series $\left\{b_{j}\right\}$ any series that satisfy:

$$
\sum_{j=0}^{\infty}\left|b_{j}\right|^{2}=1 ; b_{4 k}=b_{4 k+1}=b_{4 k+2}=b_{4 k+3} ; \quad \sum_{j=0}^{\infty}\left|\lambda_{j} b_{j}\right|^{2}=\infty
$$

and define $a_{n}=b_{n}\left(\lambda_{n} / \lambda_{n}+1 / \lambda_{n}\right)$ and adjust (2.5) by choosing the $\lambda_{n}$ :

$$
\lambda_{2 i+1}=\bar{\lambda}_{2 i} ; \quad 0<\operatorname{Re} \lambda_{4 k}=-\operatorname{Re} \lambda_{4 k+2}+1 \text {. }
$$

Thus

$$
\sum_{j=0}^{\infty} a_{j} \bar{a}_{j}=\sum_{j=0}^{\infty} b_{j} \bar{b}_{j}\left(\frac{\lambda_{j}}{\bar{\lambda}_{j}}+\frac{1}{\bar{\lambda}_{j}}\right)\left(\frac{\bar{\lambda}_{j}}{\lambda_{j}}+\frac{1}{\lambda_{j}}\right)
$$

by evaluating and using (2.4) 


$$
\begin{aligned}
& =\sum_{j=0}^{\infty} b_{j} \bar{b}_{j} \frac{\left(2 \operatorname{Re} \lambda_{j}+1\right)^{2}}{\left(2 \operatorname{Re} \lambda_{j}+1\right)\left(2 \operatorname{Re} \lambda_{j}-1\right)} \\
& =\sum_{j=0}^{\infty} b_{j} b_{j}+\sum_{j=0}^{\infty} b_{j} b_{j} \frac{2}{\left(2 \operatorname{Re} \lambda_{j}-1\right)}=1+0
\end{aligned}
$$

by (2.10), (2.11) and grouping indices $4 k$ and $4 k+2$.

Similarly

$$
\begin{aligned}
\sum_{j=0}^{\infty} a_{j} \bar{b}_{j} & =\sum_{j=0}^{\infty} b_{j} \bar{b}_{j}\left(\frac{\lambda_{j}}{\bar{\lambda}_{j}}+\frac{1}{\bar{\lambda}_{j}}\right) \\
& =\sum_{j=0}^{\infty} b_{j} \bar{b}_{j} \cdot \frac{1}{2 \operatorname{Re} \lambda_{j}-1}+1 \sum_{j=0}^{\infty} b_{j} b_{j} \cdot \frac{2 \operatorname{Im} \lambda_{j}}{2 \operatorname{Re} \lambda_{j}+1}=0 .
\end{aligned}
$$

The second sum by grouping indices $2 i$ and $2 i+1$.

Property (2.8) is valid by direct calculation.

Define now $D_{T_{0}}=D_{T_{1}^{*}}$ to be the closure in the $T$ topology of the sequence of functions:

$$
\frac{f_{n}}{\lambda_{n}}-\bar{a}_{n} \sum_{j=0}^{\infty} \frac{a_{j}}{\lambda_{j}} f_{j}-b_{n} \sum_{j=0}^{\infty} \frac{b_{j}}{\lambda_{j}} f_{j}, \quad n=0,1, \cdots .
$$

$D_{T_{0}}$ is dense in $H$, because if $\sum_{j=0}^{\infty} c_{j} f_{j}$ is orthogonal to $D_{T_{0}}$ then for every $n$ :

So

$$
\left(\sum_{j=0}^{\infty} c_{j} f_{j}, f_{n} \cdot \frac{1}{\lambda_{n}}-\bar{a}_{n} \sum_{j=0}^{\infty} \frac{a_{j}}{\lambda_{j}} f_{j}-b_{n} \sum_{j=0}^{\infty} \frac{b_{j}}{\lambda_{j}} f_{j}\right)=0 .
$$

$$
c_{n}=\bar{\lambda}_{n} a_{n} \sum_{j=0}^{\infty} \frac{\bar{a}_{j}}{\bar{\lambda}_{j}} c_{j}+\bar{\lambda}_{n} b_{n} \sum_{j=0}^{\infty} \frac{\bar{b}_{j}}{\bar{\lambda}_{j}} c_{j}
$$

which violates (2.9).

As for $\Delta_{T_{0}}$ : by (2.12) a function $\sum_{j=0}^{\infty} c_{j} f_{j}$ belongs to $\Delta_{T_{0}}^{+}$if:

$$
\left(\sum_{j=0}^{\infty} c_{j} f_{j}, f_{n}-\bar{a}_{n} \sum_{j=0}^{\infty} a_{j} f_{j}-\bar{b}_{n} \sum_{j=0}^{\infty} b_{j} f_{j}\right)=0, \quad n=0,1, \cdots .
$$

Hence $c_{n}-a_{n} \sum_{j=0}^{\infty} c_{j} \bar{a}_{j}-b_{n} \sum_{j=0}^{\infty} c_{j} b_{j}=0$. So $\Delta_{T_{i}^{*}}^{+}$(denoted also by $H_{0}^{*}$ ) is generated by the two functions $\sum_{j=0}^{\infty} a_{j} f_{j}$ and $\sum_{j=0}^{\infty} b_{j} f_{j}$. By the same calculation for $T_{1}^{*}: \Delta_{T_{1}^{*}}^{+}$or $H_{0}$ is spanned by:

$$
\sum_{j=0}^{\infty} \frac{\lambda_{j}}{\lambda_{j}} a_{j} \cdot f_{j} \text { and } \sum_{j=0}^{\infty} \frac{\lambda_{j}}{\lambda_{j}} b_{j} \cdot f_{j}
$$

The crucial step in this construction is the following relation:

$$
\left.T_{1}\left(\sum_{j=0}^{\infty} a_{j} f_{j}\right)-\sum_{j=0}^{\infty} a_{j} f_{j}=0 ; \quad T_{1}\left(\sum_{j=0}^{\infty} b_{j} f_{j}\right)\right)+\sum_{j=0}^{\infty} b_{j} f_{j}=0 .
$$


Verification of the first equality: for any $n$

$$
\begin{aligned}
& \left(\left(T_{1}-I\right) \sum_{j=0}^{\infty} a_{j} f_{j}, \frac{f_{n}}{\lambda_{n}}-\bar{a}_{n} \sum_{j=0}^{\infty} \frac{a_{j}}{\lambda_{j}} \cdot f_{j}-\bar{b}_{n} \sum_{j=0}^{\infty} \frac{b_{j}}{\lambda_{j}} f_{j}\right) \\
& =\left(\sum_{j=0}^{\infty} a_{j} f_{j},\left(\frac{\bar{\lambda}_{n}}{\lambda_{n}}-\frac{1}{\lambda_{n}}\right) f_{n}-\bar{a}_{n} \sum_{j=0}^{\infty}\left(\frac{\bar{\lambda}_{j}}{\lambda_{j}}-\frac{1}{\lambda_{j}}\right) a_{j} \cdot f_{j}-\bar{b}_{n} \sum_{j=0}^{\infty}\left(\frac{\bar{\lambda}_{j}}{\lambda_{j}}-\frac{1}{\lambda_{j}}\right) b_{j} \cdot f_{j}\right) \\
& =a_{n}\left[\left(\frac{\lambda_{n}}{\bar{\lambda}_{n}}-\frac{1}{\bar{\lambda}_{n}}\right)-\sum_{j=0}^{\infty} a_{j} \bar{a}_{j}\left(\frac{\lambda_{j}}{\bar{\lambda}_{j}}-\frac{1}{\bar{\lambda}_{j}}\right)\right]-b_{n}\left[-\sum_{j=0}^{\infty} a_{j} \bar{b}_{j}\left(\frac{\lambda_{j}}{\bar{\lambda}_{j}}-\frac{1}{\bar{\lambda}_{j}}\right)\right]
\end{aligned}
$$

by (2.6) $=-b_{n}+a_{n} \sum_{j=0}^{\infty} \bar{a}_{j} b_{j}-b_{n} \sum_{j=0}^{\infty} b_{j} \bar{b}_{j}=0$ by (2.5). The verification of the second part of (2.14) is exactly the same, except the use of (2.7) instead of (2.6).

After establishment of (2.14) the normality of $T_{0}$ is easily proved. $T_{0} T_{1}^{*}$ and $T_{1}^{*} T_{0}$ are restrictions of $T T^{*}$. So in order to prove $T_{0} T_{1}^{*}=T_{1}^{*} T_{0}$ it is sufficient to prove $\Delta_{T_{0} T_{1}^{*}}=\Delta_{T_{1}^{*} T_{0}}$ or $\Delta_{T_{0} T_{1}^{*}}^{+}=\Delta_{T_{1}^{*} T_{0}^{*}}^{+}$or to prove that the space $\hat{H}$ of functions $g$ that satisfy $T_{1} T_{0}^{*} g=0$ coincides with $\breve{H}$ the space of functions $h$ that satisfy $T_{0}^{*} T_{1} h=0$. But it is easily seen that $\hat{H}$ and $\check{H}$ are spanned by:

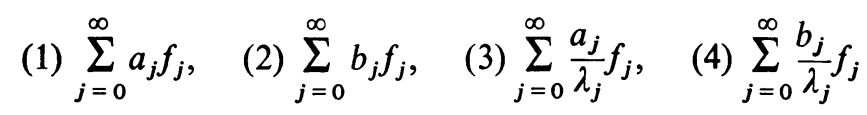

(using (2.13), (2.14) and the fact that (3) and (4) are in $D_{T}=D_{T^{*}}$ ).

Property (c) in Definition 2.1 will be established by proving that $D_{T}=D_{T_{0}^{*}}$. As $\Delta_{T}=\Delta_{T^{*}}=H$ it follows that $D_{T_{1}}=D_{T} \oplus H_{0}$ and $D_{T_{0}^{*}}=D_{T^{*}} \oplus H_{0}^{*}$. But by (2.14) $H_{0}^{*} \subset D_{T_{1}}$ and $D_{T}=D_{T^{*}}$. So $D_{T_{0}^{*}} \subset D_{T_{1}}$. In a similar way $D_{T_{1}} \subset D_{T_{0}^{*}}$.

In order to prove property (b) in Definition 2.1, it is necessary to construct in detail $D_{T_{1}^{*} T_{0}}$. By arguments similar to (2.12) $D_{T_{1}^{*} T_{0}}$ is the closure in the $T^{*} T$ norm of the sequence of functions:

$$
\frac{f_{n}}{\lambda_{n} \bar{\lambda}_{n}}-\bar{a}_{n} \sum_{j=0}^{\infty} \frac{a_{j}}{\lambda_{j} \bar{\lambda}_{j}} f_{j}-\bar{b}_{n} \sum_{j=0}^{\infty} \frac{b_{j}}{\lambda_{j} \bar{\lambda}_{j}} f_{j}-\frac{\bar{a}_{n}}{\bar{\lambda}_{n}} \sum_{j=0}^{\infty} \frac{a_{j}}{\lambda_{j}^{2} \bar{\lambda}_{j}} f_{j}-\frac{\bar{b}_{n}}{\bar{\lambda}_{n}} \sum_{j=0}^{\infty} \frac{b_{j}}{\lambda_{j}^{2} \bar{\lambda}_{j}} f_{j}
$$

Property (b) will be proved by showing that $\Delta_{T_{0}^{*}}=T \overline{D_{T_{i}^{*} T_{0}}}$. The functions $\sum_{j=0}^{\infty} c_{j} f_{j}$ that are orthogonal to $T D_{T_{1}^{*} T_{0}}$ satisfy:

$$
\left(\sum_{j=0}^{\infty} c_{j} f_{j} f_{n}-\bar{a}_{n} \sum_{j=0}^{\infty} \frac{a_{j}}{\bar{\lambda}_{j}} f_{j}-\bar{b}_{n} \sum_{j=0}^{\infty} \frac{b_{j}}{\bar{\lambda}_{j}} f_{j}-\frac{\bar{a}_{n}}{\bar{\lambda}_{n}} \sum_{j=0}^{\infty} \frac{a_{j}}{\lambda_{j} \bar{\lambda}_{j}} f_{j}-\frac{\bar{b}_{n}}{\bar{\lambda}_{n}} \sum_{j=0}^{\infty} \frac{b_{j}}{\lambda_{j} \bar{\lambda}_{j}} f_{j}\right)=0
$$

or

$$
c_{n}=\lambda_{n} a_{n} \sum_{j=0}^{\infty} \frac{\bar{a}_{j} c_{j}}{\lambda_{j}}+\lambda_{n} b_{n} \sum_{j=0}^{\infty} \frac{\bar{b}_{j} c_{j}}{\lambda_{j}}+a_{n} \sum_{j=0}^{\infty} \frac{\bar{a}_{j} c_{j}}{\lambda_{j} \bar{\lambda}_{j}}+b_{n} \sum_{j=0}^{\infty} \frac{\bar{b}_{j} c_{j}}{\lambda_{j} \bar{\lambda}_{j}} .
$$

Therefore the sequence $\left\{c_{n}\right\}$ is a linear combination in $\left\{\lambda_{n} a_{n}\right\} ;\left\{\lambda_{n} b_{n}\right\} ;\left\{a_{n}\right\} ;\left\{b_{n}\right\}$. By property (2.7) only the coefficients of the last two sequences are not zero. As $\sum_{j=0}^{\infty} a_{j} f_{j}$ and $\sum_{j=0}^{\infty} b_{j} f_{j}$ span $\Delta_{T_{0}^{*}}^{+}$the proof is complete. 
Now it is time to prove that $T_{0}$ is regular in the whole plane. $T \supset T_{0}$ and $T$ is regular at all points but $\lambda_{n}, n=0,1, \cdots$. So it is sufficient to prove that $T_{0}$ is regular at any $\lambda_{n}$.

Suppose that for some $n \lambda_{n}$ is in the spectrum of $T_{0}$. In this case there exists $g \in D_{T_{0}},\|g\|=1$ such that $\left\|\left(T-\lambda_{n} I\right) g\right\|<\varepsilon$. By the definition of $D_{T_{0}}$ (2.12) $g$ may be chosen to be of the form:

$$
\begin{aligned}
g & =\sum_{k=0}^{\infty} c_{k}\left\{\frac{b_{k}}{\lambda_{k}}-\bar{a}_{k} \sum_{j=0}^{\infty} \frac{a_{j}}{\lambda_{j}} f_{j}-\bar{b}_{k} \sum_{j=0}^{\infty} \frac{b_{j}}{\lambda_{j}} f_{j}\right\} \\
& =\sum_{j=0}^{\infty} f_{j} \cdot \frac{1}{\lambda_{j}}\left(c_{j}-a_{j} \sum_{k=0}^{\infty} c_{k} \bar{a}_{k}-b_{j} \sum_{k=0}^{\infty} c_{k} \bar{b}_{k}\right)
\end{aligned}
$$

( $g$ may be chosen so that only a finite number of the $c_{k}$ are different from zero).

Denote $A=\sum_{k=0}^{\infty} c_{k} \bar{c}_{k} ; B=\sum_{k=0}^{\infty} c_{k} \bar{b}_{k} ; d_{j}=c_{j}-a_{j} A-b_{j} B$.

$$
\begin{aligned}
\varepsilon^{2} & \geqq\left\|\left(T-\lambda_{n} I\right) g\right\|^{2}=\sum_{j=0}^{\infty}\left|\frac{\lambda_{j}-\lambda_{n}}{\lambda_{j}}\right|^{2}\left|d_{j}\right|^{2} \geqq \min _{j \neq n}\left|\frac{\lambda_{j}-\lambda_{n}}{\lambda_{j}}\right|^{2} \sum_{j \neq n}\left|d_{j}\right|^{2} . \\
\varepsilon^{2} & \geqq \sum_{j=0}^{\infty}\left|\frac{\lambda_{j}-\lambda_{n}}{\lambda_{j}}\right|^{2}\left|d_{j}\right|^{2} \geqq \min _{j \neq n}\left|\lambda_{j}-\lambda_{n}\right|^{2} \sum_{j \neq n}\left|\frac{d_{j}}{\lambda_{j}}\right|^{2} \\
& =\min _{j \neq n}\left|\lambda_{j}-\lambda_{n}\right|^{2}\left(1-\left|\frac{d_{n}}{\lambda_{n}}\right|^{2}\right) .
\end{aligned}
$$

Therefore

or:

$$
\left|d_{n}\right|^{2} \geqq\left|\lambda_{n}\right|^{2}\left(1-\frac{\varepsilon^{2}}{\min _{j \neq n}\left|\lambda_{j}-\lambda_{n}\right|^{2}}\right)
$$

$$
\frac{\left|d_{n}\right|^{2}}{\sum_{j \neq n}\left|d_{j}\right|^{2}} \geqq\left|\lambda_{n}\right|^{2}:\left(1-\frac{\varepsilon^{2}}{\min _{j \neq n}\left|\lambda_{j}-\lambda_{n}\right|^{2}}\right) \cdot \min _{j \neq n}\left|\frac{\lambda_{j}-\lambda_{n}}{\lambda_{j}}\right|^{2} \cdot \frac{1}{\varepsilon^{2}} \geqq C \cdot \frac{1}{\varepsilon^{2}}
$$

On the other hand by plugging back $c_{j}$ into $A$ and $B$ and using (2.5):

$$
d_{j}=c_{j}-a_{j} A-b_{j} B-a_{j} \sum_{k=0}^{\infty} d_{k} \bar{a}_{k}-b_{j} \sum_{k=0}^{\infty} d_{k} \bar{b}_{k} .
$$

So $a_{j} \sum_{k=0}^{\infty} d_{k} \bar{a}_{k}+b_{j} \sum_{k=0}^{\infty} d_{k} \bar{b}_{k}=0$. This equality is true for every $j$ only if $\sum_{k=0}^{\infty} d_{k} \bar{a}_{k}=0$. So

$$
\left|d_{n} \bar{a}_{n}\right|^{2}=\left|\sum_{k \neq n} d_{k} \bar{a}_{k}\right|^{2} \leqq \sum_{k \neq n}\left|d_{k}\right|^{2} \cdot \sum_{k \neq n}\left|a_{k}\right|^{2}
$$

or

which contradicts (2.16).

$$
\sum_{k \neq n} \frac{\left|d_{n}\right|^{2}}{\left|d_{k}\right|^{2}} \leqq\left|\frac{1}{a_{n}}\right|^{2}
$$


So $T_{0}$ is regular in the whole plane, and the same proof holds for $T_{1}^{*}$ as well. It remains to show that $T$ is not a polynomial in a symmetric operator. If $T=\sum_{k=0}^{m} a_{k} S^{k}$ it follows that the eigenvalues $\lambda_{n}$ of $T$ may be written as

Hence:

$$
\lambda_{n}=\sum_{k=0}^{m} a_{k} S_{n}^{k} ; S_{n} \text { real. }
$$

$$
\operatorname{Re} \lambda_{n}=\sum_{k=0}^{m} \operatorname{Re} a_{k} \cdot S_{n}^{k} \text { and } \operatorname{Im} \lambda_{n}=\sum_{k=0}^{m} \operatorname{Im} a_{k} \cdot S_{n}^{k} .
$$

The $\lambda_{n}$ satisfy (2.4):

$$
\left(\sum_{k=0}^{m} \operatorname{Re} a_{k} S_{n}^{k}\right)^{2}-\left(\sum_{k=0}^{\infty} I_{m} a_{k} \cdot S_{n}^{k}\right)^{2}=\frac{1}{2} .
$$

As this relation is true for infinitely many $S_{n}$ the following polynomial relation in a real variable $x$ is true:

$$
\left(\sum_{k=0}^{m} \operatorname{Re} a_{k} \cdot x^{k}\right)^{2}-\sum_{k=0}^{\infty}\left(\operatorname{Im} a_{k} \cdot x^{k}\right)^{2}=\frac{1}{2} .
$$

But this is true only if $\operatorname{Re} a_{k}=\operatorname{Im} a_{k}=0$ for $k \geqq 1$. Therefore $T$ is a multiple of the identity, a contradiction.

\section{Chapter 3. Extensions of Normal Operators}

Some conditions for the existence of normal extensions will be given in the first half of this chapter. The problem of necessary and sufficient conditions seems to be not an easy one, because normal operators are less restrictive than symmetric ones. Most of theorems in this direction will be for the case where $T_{0}$ and $T_{1}^{*}$ are regular in the whole plane.

The second half will deal with properties of the spectrum of normal extensions. In particular the distribution of the eigenvalues of complete normal extensions will be investigated.

Definition 3.1. $\tilde{T}$ an extension of $T_{0}$ is a normal extension if $\tilde{T}$ is normal.

DEFINITION 3.2. $\tilde{T}$ a normal extension of $T_{0}$ is a complete normal extension regular in the neighborhood of zero if $\Delta_{\tilde{T}}=H$ and $\tilde{T}^{-1}=R$ is a bounded normal operator

It is easy to produce examples of extensions (in the sense of Chapter 1) of normal operators which are not normal. Any nonsymmetric extension of a symmetric operator is of this type.

As for normal extensions and, especially, complete normal extensions, the following theorems will be based on the reasoning below (in the case where $T_{0}$ and $T_{1}^{*}$ are regular in the whole plane): It is known by a theorem of G. C. Rota [4] that the spectrum of a regular extension (not necessarily normal) is composed of a denumerable number of isolated points. By virtue of Definition 3.2, if $\lambda$ is a 
point in the spectrum of $T$ then there exists a function $f$ such that $T f=\lambda f, T^{*} f=\lambda f$. But $T \subset T_{1}$ and $T^{*} \subset T_{0}^{*}$; therefore $T_{1} f=\lambda f ; T_{0}^{*} f=\lambda f$. Theorem 2.3 may be utilized now to get:

THEOREM 3.1. $\left(T_{0}, T_{1}^{*}\right)$ form a normal pair of operators regular in the whole plane and having a finite deficiency index.

A necessary condition for existence of complete normal extensions is existence of solutions to the equation: $Q(\lambda, \lambda)=0$.

THEOREM 3.2. Subject to the conditions of Theorem 3.1, all the eigenvalues of a complete normal extension are on the manifold of real zeros of the polynomial in $\mu$ and $v: Q(\mu+i v, \mu-i v)=0$.

In a very special case the following necessary and sufficient condition is obtained:

THEOREM 3.3. If in addition to the conditions of Theorem $3.1 T_{0}$ and $T_{1}^{*}$ have the deficiency index 1 then a necessary and sufficient condition for existence of complete normal extensions of $T_{0}$ is: There exists a symmetric operator $S_{0}$ such that $T_{0}=\gamma S_{0}+\delta I$.

Proof. If $T_{0}=\gamma S_{0}+\delta I$ and $T_{0}$ is regular in the whole plane, then $S_{0}$ is also regular in the whole plane; therefore $S_{0}$ has a self-adjoint extension $S$. Define now $T=\gamma S+\delta I$ to get the desired extension of $T_{0}$.

Suppose now that there exists a complete normal extension $T$ of $T_{0}$. In this case there exists a sequence of eigenfunctions $\left\{f_{n}\right\}$ such that:

$$
T f_{n}=\lambda_{n} f_{n} ; T^{*} f_{n}=\lambda f_{n} .
$$

As $T_{1} \supset T$ and $T_{0}^{*} \supset T^{*}$ it follows from Theorem 2.4 that

$$
\bar{\lambda}_{n}=\alpha \lambda_{n}+\beta
$$

Denote $\lambda_{n}=\mu_{n}+i v_{n}$ and separate to real and imaginary parts to obtain

$$
\begin{aligned}
& (\operatorname{Re} \alpha-1) \mu_{n}-\operatorname{Im} \alpha \cdot v_{n}+\operatorname{Re} \beta=0, \\
& \operatorname{Im} \alpha \cdot \mu_{n}+(\operatorname{Re} \alpha+1) v_{n}+\operatorname{Im} \beta=0 .
\end{aligned}
$$

Define $S$ by:

$$
S=\frac{T-\left(\mu_{0}+i v_{0}\right) I}{\operatorname{Im} \alpha+i(\operatorname{Re} \alpha-1)}
$$

$\mathrm{S}$ is symmetric because $S f_{n}=t_{n} f_{n}$ and $t_{n}$ is real. Because by a direct compuation:

$$
S f_{n}=\frac{\mu_{n}+i v_{n}-\left(\mu_{0}+i v_{0}\right)}{\operatorname{Im} \alpha+i(\operatorname{Re} \alpha-1)} f_{n}=t_{n} f_{n}
$$

and using (3.1) it follows that $\operatorname{Im} t_{n}=0$. 
Remark. This theorem explains the choice of deficiency index 2 in the example in Chapter 2.

The difference between normal and symmetric operators is emphasized by the following theorems.

THEOREM 3.4. $T_{0}$ is a normal operator regular in the whole plane and having a finite deficiency index $n$. A necessary condition for existence of complete normal extension is: There exist $n$ independent eigenfunctions $f_{j}$ independent $\left(\bmod \Delta_{T_{0}}\right)$ such that:

$$
T_{1} f_{j}=\lambda_{j} f_{j} ; T_{0}^{*} f_{j}=\lambda_{j} f_{j} ;\left(f_{i}, f_{j}\right)=\delta_{i j} .
$$

Proof. If $T_{0}$ has complete normal extension $T$ then there exists an orthonormal sequence of eigenfunctions $\left\{f_{i}\right\}$ such that $T_{1} f_{i}=\lambda_{i} f_{i} ; T_{0}^{*} f_{i}=\bar{\lambda}_{i} f_{i}$. As $\Delta_{T}=H$ it follows that $n$ eigenfunctions may be picked out of the $f_{i}$ which together with $\Delta_{T_{0}}$ span $H$.

In the symmetric case

THeOReM 3.5. If $T_{0}$ is symmetric then $T_{0}-\lambda I(\operatorname{Im} \lambda \neq 0)$ is normal. Any self-adjoint extension of $T_{0}$ induces a complete normal extension of $T-\lambda I$.

If $T_{0}$ is regular in the whole plane then the conditions in Theorem 3.4 are sufficient and any complete normal extension is self-adjoint.

Proof. The first part is obvious. As for the second part: In the symmetric case $T_{0}=T_{1}^{*}$ and $T_{1}=T_{0}^{*}$. Therefore any common eigenvalue $z$ such that $T_{1} f=z f$; $T_{0}^{*} f=\bar{z} f$ is real, hence the extension is self-adjoint.

For later application the following is needed.

THEOREM 3.6. $T_{0}$ is symmetric regular operator. $M$ is defined to be the manifold of all forthogonal to $\Delta_{T_{0}}\left(M \perp \Delta_{T_{0}}\right) . T$ is an extension defined by:

$$
D_{T}=D_{T_{0}} \oplus\left\{f_{\alpha}\right\} ; \quad T f=0, \quad f \in M
$$

Conclusion: $T$ is self-adjoint.

Proof. $T$ is symmetric because if $h_{1}, h_{2} \in D_{T_{0}}$ and $f_{1}, f_{2}, \in \Delta_{T_{0}}$ then:

$$
\left(T\left[h_{1}+f_{1}\right], h_{2}+f_{2}\right)=\left(T h_{1}, h_{2}\right)=\left(h_{1}, T h_{2}\right)=\left(h_{1}+f_{1}, T\left[h_{2}+f_{2}\right]\right) .
$$

$T$ is self-adjoint: Any $g$ such that $T^{*} g=0$ satisfies: $0=\left(T^{*} g, h\right)=(g, T h)$ or $g \perp \Delta_{T_{0}}$ hence $g \in D_{T}$. Moreover $\Delta_{T^{*}}=\Delta_{T_{0}}$ because for any $f \perp: \Delta_{T_{0}}$ : $\left(T^{*} g, f\right)=(g, T f)=0$. So any $g \in D_{T^{*}}$ may be decomposed into $g_{1}+g_{2}: g_{1} \perp \Delta_{T_{0}}$, $g_{2} \in D_{T_{0}}$. Thus the proof of the theorem is complete.

Passing now to properties of complete normal extensions the following theorems will deal mainly with local as well as asymptotic distribution of the eigenvalues. These theorems are a direct consequence of normality and are not valid for general operators. 
THEOREM 3.7. $T_{0}$ is a normal operator regular in $\lambda$ satisfying: $\left\|T_{0} f-\mu f\right\| \geqq c\|f\|$ for $|\mu-\lambda|<d$ and having deficiency index $n . T$ is a complete normal extension regular at $\lambda$ (some point in a neighborhood of $\lambda$ ). $\psi_{1}, \cdots, \psi_{n+1}$ are $n+1$ linearly independent functions which satisfy $T \psi_{i}=\mu_{i} \psi_{i}$.

Subject to these conditions: $\max _{i}\left|\mu_{i}-\lambda\right| \geqq \min \{d, c\}$.

Proof. The theorem is interesting only in the case where all the $\mu_{i}$ satisfy $\left|\mu_{i}-\lambda\right|<d$. Without loss of generality $\lambda$ may be set to be zero and $\psi_{i}$ to be orthnormal. As the deficiency index is $n$ there exists a linear combination $\sum_{i=1}^{n+1} a_{i} \psi_{i}=f$ such that $f \in \Delta_{T_{0}}$. If for some $i: T \psi=0$ : then $a_{i}=0$ as well, because as $T$ is normal $T \psi_{i}=0$ implies $T^{*} \psi_{i}=0$ or $T_{0}^{*} \psi_{i}=0$ so $\psi_{i} \perp \Delta_{T_{0}}$.

Hence as $T^{-1}$ is a well-defined operator from $\Delta_{T_{0}}$ to $H$ :

$$
\begin{aligned}
\left\|T^{-1} f\right\|^{2} & =\left\|\sum_{s} a_{s} T^{-1} \psi_{s}\right\|^{2}=\sum_{s}\left|\frac{a_{s}}{\mu_{s}}\right|^{2} \geqq \min _{s} \frac{1}{\left|\mu_{s}\right|^{2}} \cdot \sum_{s}\left|a_{s}\right|^{2} \\
& =\min _{s} \frac{1}{\left|\mu_{s}\right|^{2}}\|f\|^{2} .
\end{aligned}
$$

On the other hand as $f \in \Delta_{T_{0}}:\|f\|^{2} \geqq c^{2}\left\|T^{-1} f\right\|^{2}$. Hence

$$
\frac{\|f\|^{2}}{c^{2}} \geqq\left\|T^{-1} f\right\|^{2} \geqq \min _{s} \frac{1}{\left|\mu_{s}\right|^{2}}\|f\|^{2}
$$

or $\max _{i}\left|\mu_{i}\right| \geqq c$.

In certain special cases (conditions of Theorem 3.6, for example), a better estimate holds.

THEOREM 3.8. $T_{0}$ is a normal operator regular in $\lambda$ satisfying: $\left\|T_{0} f-\mu f\right\| \geqq c\|f\|$ for $|v-\lambda|<d$ (having any deficiency index); $T$ is a complete normal extension which is constructed by adjoining all the eigenfunctions of $T_{0}$ which have eigenvalue $\lambda$; $\phi$ is any eigenfunction of $T$ such that $T \phi=\mu \phi, \mu \neq \lambda_{0}$.

Subject to these conditions $|\mu-\lambda| \geqq \min \{d, c \sqrt{ } 2\}$.

Proof. The construction above does not necessarily lead to a complete normal extension. In the case it does, assuming $\lambda=0, \phi$ is orthogonal to any $\phi_{\alpha}$ satisfying $T \phi_{\alpha}=0$ or, by the theorem's conditions it is orthogonal to any $\psi: T_{0}^{*} \psi=0$. Hence

$$
\phi=T_{0} f \text { and } \phi-\mu f=T_{0} f-\mu f .
$$

Separate $f$ into components $f_{1}$ in the direction of $\phi, f_{2}$ orthogonal to $\phi$. As $T$ is complete normal extension $\phi$ is an eigenfunction of $T$ satisfying $T^{*} \phi=\bar{\mu} \phi$ or $\phi$ orthogonal to $\Delta_{T_{0}}=\mu I$. Therefore

$$
T_{0} f-\mu f=-\mu f_{2} ; \quad T_{0} f=\phi=\mu f_{1} .
$$


But

$$
\begin{aligned}
& c^{2}\|f\|^{2} \leqq\left\|T_{0} f\right\|^{2}=|\mu|^{2}\left\|f_{1}\right\|^{2}, \\
& c^{2}\|f\|^{2} \leqq\left\|T_{0} f-\mu f\right\|^{2}=|\mu|^{2}\left\|f_{2}\right\|^{2} .
\end{aligned}
$$

So by addition $2 c^{2}\|f\|^{2} \leqq|\mu|^{2}\left\{\left\|f_{1}\right\|^{2}+\left\|f_{2}\right\|^{2}\right\}=|\mu|^{2}\|f\|^{2}$ which is the desired result.

THEOREM 3.9. $T_{0}$ is a normal operator regular in the whole plane and having a finile deficiency index. $T$ is any complete normal extension of $T_{0}$.

Subject to these conditions there is only a finite number of directions $\alpha_{1}, \cdots, \alpha_{m}$ having the property: For any $\varepsilon$ there exists an $n$ such that all the eigenvalues $\lambda_{i}$ $(i \geqq n)$ of $T$ are located in the angles bounded by $\alpha_{j}-\varepsilon ; \alpha_{j}+\varepsilon$.

Proof. By Theorem 3.7 there is only a finite number of eigenvalues (counting multiplicity) in any bounded portion of the plane. Therefore it is sufficient to investigate the asymptotic properties of the zero-manifold of the polynomial $Q(x, y)$ (Theorem 3.2). So by proving the following lemma, the theorem will be established.

LEMma 3.1. Any unbounded analytic curve which is included in the manifold of real zeros of a polynomial in two variables $Q(x, y)$ has an asymptotic direction $\alpha$, i.e., for any $\varepsilon$ there exists an $R$ having the property: The direction of the tangent at $(x, y), x^{2}+y^{2} \geqq R^{2}$, differs from $\alpha$ by $\varepsilon$ at most.

Proof. It is well known that the manifold of the real zeros is composed of a finite number of points and analytic curves having a finite number of singular points. Choose $R_{0}$ so that the only singular point outside a circle with radius $R_{0}$ about the origin is infinity. Any analytic curve outside this circle is unbounded. Take one of these curves and denote it by $y(x)$. Denote $z=x+i y$ and introduce new variables by inversion $1 / z=\zeta=\xi+i \eta$. By this inversion the real zeros of $Q(x, y)$ correspond to the real zeros of a polynomial $P(\xi, \eta)$. In particular $y(x)$ corresponds to an analytic curve $\eta(\xi)$ regular inside a circle of radius $1 / R_{0}$ which has a singularity at zero (at most). Any tangent to $y(x)$ will correspond to a tangent circle to $\eta(\xi)$ passing through the origin.

Denote by $S(\xi)$ the tangent circle at $(\xi, \eta(\xi))$. By conformity it is sufficient to prove that there exists a limit to the angle between the $\xi$ axis and the tangent to the circle at the origin.

Denote by $(\xi, \eta)$ the center of $S(\xi)$, then:

$$
(\xi-\hat{\xi})^{2}+(\eta-\hat{\eta})^{2}=\hat{\xi}^{2}+\hat{\eta}^{2} \quad-\frac{\xi-\hat{\xi}}{\eta-\hat{\eta}}=\eta^{\prime}(\xi) .
$$

By these two equations $\hat{\xi}$ and $\hat{\eta}$ are algebraic in $\xi, \eta(\xi)$ and $\eta^{\prime}(\xi)$. As $\eta(\xi)$ and $\eta^{\prime}(\xi)$ 
can be developed by Puissex series (fractional power series) and $\eta(\xi)$ is developed only by positive powers $(\eta=0$ when $\xi=0$ ) the functions $\xi(\xi), \hat{\eta}(\xi)$ can be developed to the series:

$$
\begin{array}{ll}
\hat{\xi}(\xi)=\xi^{\alpha} \sum_{i=0}^{\infty} a_{i} \xi^{i / k}, & a_{0} \neq 0 \text { and } k \text { integer, } \\
\hat{\eta}(\xi)=\xi^{\beta} \sum_{i=0}^{\infty} b_{i} \xi^{i / l}, & b_{0} \neq 0 \text { and } l \text { integer. }
\end{array}
$$

Therefore $\hat{\xi}(\xi) / \hat{\eta}(\xi)$ is close to $\left(a_{0} / b_{0}\right) \xi^{\alpha-\beta}$ as $\xi \rightarrow 0$. But $\hat{\xi}(\xi) / \hat{\eta}(\xi)$ is the angle between the circle and the $\xi$ axis, therefore the limit exists (which may be infinite) and the lemma is proved.

From these theorems one can draw conclusions about the operator $T^{-1}$ and to investigate its type, i.e., if it is compact or of Hilbert-Schmidt type, etc.

Definition 3.3. $\lambda$ will be called an essential eigenvalue if there exists a function $\psi$ (essential eigenfunction) such that $\psi \in D_{T_{0}}$ and $T_{0} \psi=\lambda \psi$.

THEOREM 3.10. $T$ is a complete normal extension of $T_{0}$ regular at 0 . A necessary condition for $T^{-1}$ to be compact is:

(a) There is only finite number of eigenvalues $\lambda_{i}$ (counting multiplicity): $\alpha \leqq \lambda_{i} \leqq \beta$ for any $\alpha$ and $\beta$.

(b) $\hat{T}_{0}$, the operator $T_{0}$ restricted to the orthogonal complement of the essential eigenfunctions, is regular in the whole plane.

Proof. (a) is self evident. As for (b) suppose that there exists a point $\mu$ such that $\hat{T}_{0}-\mu I$ is not regular. In this case there are three possibilities:

(i) $\mu$ is in the continuous spectrum of $T$.

(ii) $\mu$ is an eigenvalue having infinite multiplicity.

(iii) $\mu$ is an eigenvalue having finite multiplicity.

As the first two possibilities contradict the compactness of $T^{-1}$, it remains to consider only the third one.

$T_{0}-\mu I$ is not regular; therefore there exists a sequence $\left\{f_{n}\right\} \in D_{\hat{T}_{0}}\left\|f_{n}\right\|=1$ such that $\left\|\hat{T}_{0} f_{n}-\mu f_{n}\right\| \leqq \varepsilon_{n}, \varepsilon_{n} \rightarrow 0$. As $\mu$ is an isolated point in the spectrum of $T$ any $f_{n}$ can be decomposed to orthogonal components:

$$
f_{n}=f_{n, 1}+f_{n, 2} ; T f_{n, 1}=\mu f_{n, 1} ;\left\|T f_{n, 2}-\mu f_{n, 2}\right\| \geqq \alpha\left\|f_{n, 2}\right\| .
$$

Therefore

$$
\begin{aligned}
\varepsilon_{n} & =\varepsilon_{n}\left\|f_{n}\right\| \geqq\left\|\hat{T}_{0} f_{n}-\mu f_{n}\right\|=\left\|T f_{n, 1}-\mu f_{n, 1}+T f_{n, 2}-\mu f_{n, 2}\right\| \\
& =\left\|T f_{n, 2}-\mu f_{n, 2}\right\| \geqq \alpha\left\|f_{n, 2}\right\| .
\end{aligned}
$$

So

$$
f_{n, 2} \rightarrow 0 \quad \text { as } n \rightarrow \infty
$$


The functions $f_{n, 1}$ are bounded by 1 and as they are in a finite subspace, a strongly convergent subsequence may be chosen out of them. For sake of simplicity keep the same index. As $f_{n}=f_{n, 1}+f_{n, 2}$ is convergent to $f$ and $\hat{T}_{0} f_{n}=T f_{n, 1}+T f_{n, 2}$ converges to $\mu f, D_{\widehat{T}_{0}}$ contains an essential eigenvalue, contrary to the assumptions.

As for sufficient condition the following holds.

THEOREM 3.11. Subject to the conditions of Theorem 3.10 a sufficient condition for $T^{-1}$ to be compact is: $\hat{T}_{0}$ is regular and has a finite deficiency index in the whole plane.

Proof. In this case the spectrum is composed of denumerable number of eigenvalues. Moreover Theorem 3.9 assures that the multiplicity of any eigenvalue is finite (does not exceed the deficiency index).

In case of infinite deficiency index there are examples of compact as well as noncompact inverses of a complete normal extension.

In some special cases where $T_{0}$ is regular in the whole plane the lower bound of $T_{0}-\lambda I$ is known (the greatest $C(\lambda)$ satisfying $\left\|T_{0} f-\lambda f\right\| \geqq C(\lambda) f$ ). For example, the Hörmander inequality [2] leads to the estimate $\|T f-\lambda f\| \geqq C\|f\|$, independent of $\lambda$. In these cases the combination of Theorem 3.9 and Lemma 3.1 leads to estimates of the distribution of the eigenvalues.

Definition 3.4. $S$ a bounded operator in Hilbert space is of abstract HilbertSchmidt type if it has the property:

$$
\|S\| \mid-\sum_{\alpha \in J}\left\|S \phi_{\alpha}\right\|^{2}<\infty
$$

where $\left\{\phi_{\alpha}\right\}_{\alpha \in J}$ is a complete orthonormal system.

||$|S|||$ is called the double norm of $S$ and is independent of $\phi_{\alpha}$. In case of Hilbert space of functions this definition coincides with the known one:

$$
S f(t)=\int K(s, t) f(t) d t=g(s) ; \quad \iint|K(s, t)|^{2} d s d t<\infty
$$

( $K$ is not necessarily symmetric). A Hilbert-Schmidt operator is always compact and satisfies: $\Sigma\left|\lambda_{n}\right|^{2}<\infty$ where $\left\{\lambda_{n}\right\}$ is the sequence of eigenvalues (counting multiplicity).

A wider class is that of operators satisfying $\sum_{i}\left|\lambda_{i}\right|^{l}<\infty$. Any of these operators has the property that a certain power of it (the [l/2]) is of Hilbert-Schmidt type.

THEOREM 3.12. $T_{0}$ is a normal operator regular in the whole plane having a finite deficiency index, and satisfying:

$$
\left\|T_{0} f-\lambda f\right\| \geqq C(\lambda)\|f\| \text { for } f \in D_{T_{0}} .
$$

$T$ is a complete normal extension regular at zero.

Define: 


$$
M(R)=\min _{|\lambda|=R} C(\lambda) .
$$

Conclusion: If $M(R)>R^{-k+8}$ for $R$ sufficiently large then $T^{-1}$ is a compact operator having the property $\Sigma\left|\mu_{i}\right|^{1+k}<\infty ; \mu_{i}$ are the eigenvalues of $T^{-1}$, counting multiplicity.

COROLlaRY 3.12. If $k=1$ then $T^{-1}$ is of abstract Hilbert-Schmidt type.

The proof of the theorem will be via the following lemmas.

LEMMA 3.2. $M(R)$ is a positive continuous function of a real variable which satisfies $M(R)>R^{-k+\varepsilon}$ for $R$ sufficiently large. $\left\{\eta_{i}\right\}$ is an increasing sequence of positive numbers satisfying: $\eta_{i+1}-\eta_{i} \geqq M\left(\eta_{i}\right)$. Conclusion: $\sum_{i=1}^{\infty}\left(1 / \eta_{i}\right)^{l}<\infty$ where $l=\max \{2, k+1\}$.

Proof. If $k<1$ it may be replaced by 1 in the lemma's condition so $l$ may be replaced by $k+1 ;\left\{\eta_{i}\right\}$ is an isolated sequence, so it is sufficient to prove the convergence by summing from some $\eta_{m}>1$. For sake of simplicity take $\eta_{1}>1$.

It is sufficient to prove $\eta_{i}^{k+1} \geqq(i-1)^{1+\delta}$ where $\delta$ is some positive number to be fixed. The proof will be by induction. Distinguish between two cases.

$$
\text { (a) } \eta_{i}^{k+1} \geqq i^{1+\delta}, \quad \text { (b) } \eta_{i}^{k+1}<i^{1+\delta} \text {. }
$$

In case (a) as $\eta_{i+1}>\eta_{i}$ it follows that $\eta_{i+1}^{k+1} \geqq i^{1+\delta}$ which is the induction hypothesis.

In case (b) it is sufficient to show: $(i+1)^{1+\delta}-\eta_{i+1}^{k+1} \leqq i^{1+\delta}-\eta_{i}^{k+1}$. Because as $(i+1)^{1+\delta}-i^{1+\delta}>i^{1+\delta}-(i-1)^{1+\delta}$ it follows that

$$
i^{1+\delta}-\eta_{i+1}^{k+1} \leqq(i-1)^{1+\delta}-\eta_{i}^{k+1} \leqq 0 .
$$

The inequality above reduces to:

$$
(i+1)^{(1+\delta) /(1+k)}-\eta_{i+1} \leqq i^{(1+\delta) /(1+k)}-\eta_{i}
$$

or

$$
\begin{array}{r}
\eta_{i+1}-\eta_{i} \geqq(i+1)^{(1+\delta) /(1+k)}-i^{(1+\delta) /(1+k)} . \\
\eta_{i}+M\left(\eta_{i}\right)-\eta_{i} \geqq(i+1)^{(1+\delta) /(1+k)}-i^{(1+\delta) /(1+k)}
\end{array}
$$

or

$$
\eta_{i}^{-k+\varepsilon} \geqq i^{(1+\delta) /(1+k)} \quad\left[\left(1+\frac{1}{i}\right)^{(1+\delta) /(1+k)}-1\right] .
$$

So applying the mean value theorem it reduces to:

$$
\eta_{i}^{-k+\varepsilon} \geqq i^{(1+\delta) /(1+k)} \cdot \frac{1+\delta}{1+k} \cdot \frac{1}{i} \cdot\left(1+\frac{\theta}{i}\right)^{(1+\delta) /(1+k)-1} \quad(0 \leqq \theta \leqq 1) .
$$


Or by the induction hypotheses:

$$
\left(i^{(1+\delta) /(1+k)}\right)^{-k+\varepsilon} \geqq i^{(1+\delta) /(1+k)} \cdot \frac{1+\delta}{1+k} \cdot \frac{1}{i} \cdot\left(1+\frac{\theta}{i}\right)^{(1+\delta) /(1+k)-1} .
$$

As for small $\delta$ :

$$
\frac{1+\delta}{1+l k} \cdot\left(1+\frac{\theta}{i}\right)^{(1+\delta) /(1+k)-1} \leqq 1
$$

And for $\delta \leqq \varepsilon /(1+k-\varepsilon)$

$$
\frac{1+\delta}{1+k}(-k+\varepsilon) \geqq \frac{1+\delta}{1+k}-1
$$

the last inequality is valid which completes the proof.

LemMa 3.3. $y(x)$ is an unbounded curve having the property: For any $\varepsilon$ there exists a constant $R_{0}$ such that the direction of the tangent to $y(x)$ : $y^{2}+x^{2} \geqq R_{0}^{2}$ differs from a fixed direction $\alpha$ by $\varepsilon$ at most. Then there exists $a$ cons ant $R$ having the property: The distance between any two points on the curve: $\left(x_{1}, y\left(x_{1}\right)\right) ;\left(x_{2}, y\left(x_{2}\right)\right): y_{i}^{2}+x_{i}^{2} \geqq R^{2}$ is comparable with the difference of their distance from the origin, i.e.:

$$
\left|\frac{\left(x_{2}-x_{1}\right)^{2}+\left[y\left(x_{2}\right)-y\left(x_{1}\right)\right]^{2}}{x_{2}^{2}+y\left(x_{2}\right)^{2}-\left(x_{1}^{2}+y\left(x_{1}\right)^{2}\right)^{2}}\right| \geqq \frac{1}{2} .
$$

Proof. By direct verification.

Proof of Theorem 3.12. It is known from Theorem 3.9 that there is only a finite number of eigenvalues in any bounded set in the plane, so it is sufficient to consider the sum of eigenvalues outside a large disc. All the eigenvalues are on the real-zeros manifold of a polynomial in two variables. Consider the disc that outside it this manifold satisfies the conclusions of Lemma 3.1. This manifold contains $m$ analytic curves; each of them satisfies the conditions of Lemma 3.3. Therefore there exists a constant $R$ satisfying the conclusions of this lemma for every curve.

Denote by $n$ the deficiency index of $T_{0}$ and order the eigenvalues $\lambda_{i}$ according to their absolute value (counting multiplicity). Consider now any block of eigenvalues having $m n+1$ terms, i.e., the sequence $\lambda_{i}$ for $l \leqq i<l+m n+1$. There exists at least one curve which contains $n+1$ eigenvalues, and by Theorem 3.9, there exist at least two out of these eigenvalues satisfying:

$$
\left|\lambda_{i_{2}}-\lambda_{i_{1}}\right| \geqq M\left(\left|\lambda_{i_{1}}\right|\right)
$$

hence by Lemma 3.3:

$$
\left|\lambda_{i_{2}}\right|-\left|\lambda_{i_{1}}\right| \geqq \frac{M\left(\left|\lambda_{i_{1}}\right|\right)}{2}
$$

and by monotonicity: 


$$
\left|\lambda_{l+m n+1}\right|-\left|\lambda_{k}\right| \geqq \frac{M\left(\left|\lambda_{i_{1}}\right|\right)}{2} \geqq\left|\lambda_{i_{1}}\right|^{-k+\varepsilon} \geqq\left|\lambda_{l}\right|^{-k+\varepsilon} \text {. }
$$

Separate now the sequence $\left\{\left|\lambda_{i}\right|\right\}$ to $m n$ sequences

$$
\left\{\left|\lambda_{s+t m n}\right|\right\}, \quad s=1, \cdots, m n ; t=1, \cdots, \infty .
$$

Any of these sequences satisfies the conditions of Lemma 3.2, therefore satisfies its conclusions and by grouping these sequences the theorem is proved as well.

\section{REFERENCES}

1. P. D. Lax, On Cauchy's problem for hyperbolic equations and the differentiability of solutions of elliptic equations, Comm. Pure Appl. Math. 8 (1955), 615-633.

2. L. Hörmander, On the theory of general partial differential operators, Acta Math. 94 (1955), 161-248.

3. N. I. Achiezer and I. M. Glasman, Theorie der linearen Operatoren im Hilbert-Raum, Akademie-Verlag, Berlin, 1954.

4. G. C. Rota, Extension theory of differential operators. I, Comm. Pure Appl. Math. 11 (1958), 23-65.

5. I. C. Gohberg and M. G. Krein, Systems of integral equations on a half line with kernels depending on the difference of arguments, Amer. Math. Soc. Transl. (2) 14 (1960), 217-287.

6. F. Riesz and B. Sz. Nagy, Functional analysis, Ungar, New York, 1955.

The Hebrew University, JERUSALEM, ISRAEL

The University of Chicago, Chicago, Illinors 